\title{
Somatic Dysfunctions of Hip and Pelvis Overlooked in a Case of Vulvodynia
}

Athina Giovanis, DO; Stephanie Zeszutek, DO

From the Department of Osteopathic Medicine (Dr Giovanis) and the Department of Primary Care (Dr Zeszutek) at Touro College of Osteopathic Medicine in Middletown, New York.

Financial Disclosures: None reported.

Support: None reported.

Address correspondence to Athina Giovanis, DO, Department of Osteopathic Medicine at Touro College of

Osteopathic Medicine 60 Prospect Ave, Room 203, Middletown, NY 10940-4133. athina.giovanis@touro.edu

Submitted January 14, 2020; final revision received July 22, 2020; accepted July 31, 2020.
Vulvodynia is chronic perineal pain in women. Repercussions of this disorder can have a negative effect on women's health and lifestyle. The origin is often multifactorial, including pelvic and lower extremity somatic dysfunctions. If left untreated, these somatic dysfunctions can directly alter ligamentous tension on the pelvic floor and surrounding regions, resulting in perineal pain. Management of vulvodynia must be individualized due to the multifactorial etiology and complicated structure and function of the pelvic floor muscles. The authors present a case of vulvodynia in which osteopathic manipulative treatment was an effective management technique.

J Am Osteopath Assoc. 2020;120(11):792-795. Published online October 6, 2020. doi:10.7556/jaoa.2020.140

Keywords: osteopathic manipulative treatment, vulvodynia<smiles>[AlH2]</smiles>
ulvodynia is chronic perineal pain in women. In 2014, $16 \%$ of women of reproductive age in the United States were diagnosed with vulvodynia. ${ }^{1,2}$ Due to the sensitive nature of vulvodynia and its impact on psychological and sexual function, the incidence is likely underreported. Many women never seek care, and those who do often do not receive a diagnosis of vulvodynia. ${ }^{1,2}$ Vulvodynia can present with nonspecific symptoms, including burning and irritation for more than 3 months. Pain can present spontaneously or after provocation and can be localized to 1 area of the vulva or generalized to the entire vulva. ${ }^{1}$ Vulvodynia appears to be a multifactorial disorder from a wide range of potential origins, including lumbosacral pathology, frequent vaginal infections, neuropathic pain, pelvic floor hypertonicity, pelvic organ prolapse, hormone imbalances, and psychological disorders. ${ }^{1,3,4}$ Vulvodynia tends to lead to pelvic floor/ diaphragm dysfunction that results in pelvic floor hypertonicity or high resting tension, tenderness to palpation, and weakness. ${ }^{5}$ Somatic dysfunction (SD) of the pelvis and lower limbs can directly alter pelvic floor mechanics. Injuring the pelvis alters sacral mechanics and leads to sacral ligamentous dysfunction affecting the pelvic floor. Chronic hip dysfunction is often associated with a sacroiliac (SI) joint dysfunction, which also alters the function of the pelvic diaphragm. When unaddressed, these dysfunctions may result in vulvodynia. ${ }^{6}$

Various management techniques are used for vulvodynia pain, such as medications, physiotherapy, and injections. Clinically, patients often have limited or no response to these treatment modalities. ${ }^{1,5}$ Osteopathic manipulative treatment (OMT) should be considered when treating patients with vulvar pain, as the basic osteopathic principles, "the body is a unit" and "structure and function are reciprocally interrelated," are addressed. Open communication is important to keep the patient engaged in her care. Clear, informed consent for every noninvasive or invasive procedure is critical for a 
healthy patient-physician relationship, as well as ensuring the patient feels empowered throughout her health care journey. ${ }^{7,8}$

\section{Anatomy Overview}

The bony structure of the pelvis is formed by the innominate bones that meet anteriorly at the pubic symphysis. The sacrum articulates within the innominate bones posteriorly and the coccyx inferiorly. The thoracolumbar fascia (TLF) is dense tissue that extends from the sacrum to the upper back and neck. The 3 connective tissue layers of the TLF enclose the erector spinae, multifidus, and quadratus lumborum muscles. Muscle tone within the TLF increases fascial tension, thus increasing ligamentous tension in the deep sacroiliac (SI) joint. ${ }^{9-11}$ The form and force closure of the interlocking joint surfaces and the tension provided by the TLF help stabilize the pelvis. Additionally, the contractile forces of the levator ani and coccygeus muscles (or pelvic diaphragm), which attach anteriorly on the sacrum, function during respiration to maintain pelvic organ support. ${ }^{10,12}$ The muscles, ligaments, and fascia stabilize the pelvis and act as a pelvic floor sling to support the bladder, reproductive organs, and rectum. ${ }^{3}$

The stability of the pelvic girdle also depends on coordinated tone and activation of the surrounding myofascial components, such as the lower limb. ${ }^{13}$ The TLF is linked via the gluteus maximus to the fascia lata, which encases the hamstrings, quadriceps, and adductors. ${ }^{9}$ Additionally, the sacrotuberous ligament is continuous with the ischium, the fascial sheath of the pudendal nerve, the lowest fiber of the gluteus maximus, and the biceps femoris. ${ }^{10,14}$ Increased tension in the biceps femoris alters sacrotuberous ligament tension, which affects SI joint function. ${ }^{15}$ Ultimately, dysfunction of sacral motion alters ligamentous tension on the uterus or the pelvic floor and can result in perineal pain. ${ }^{6,16}$

Perineal pain is mediated via the inferior hypogastric plexus (IHP), a paired structure lateral to the uterine cervix innervating the pelvic viscera. The IHP is com- posed of postganglionic sympathetic, preganglionic and postganglionic parasympathetic, and visceral afferent fibers. The sympathetic fibers of the IHP mediate nociceptive sensations from the viscera. If the pain input originates superior to the cervix, it will enter the spinal cord via the superior hypogastric plexus and show signs of sensitization at T10 to T12. Pain at or below the cervix will enter the sacrum resulting in low back pain. Pudendal afferent nerve entrapment can also cause pelvic floor pain. ${ }^{17}$

\section{Report of Case}

A 40-year-old woman, referred for osteopathic evaluation by her gynecologist, presented with complaints of perineal pain that began about a year earlier. Initially, she experienced left hip, hamstring, and buttock pain after a vigorous walk. She then developed pelvic floor pain that became a constant ache, and sexual intercourse became painful. Pain in her left hip and pelvic floor worsened when lying on her left side or with prolonged sitting. A cystoscopy and vaginal ultrasonography were normal. Management consisted of 6 months of physical therapy (PT), including ultrasound therapy, dry needling, an SI support belt, and a heel lift on the left. For the last 3 months, PT specifically focused on the pelvic floor was added as part of her treatment. Due to limited treatment response, she had 3 trigger point injections and 4 vulvar Botox injections to manage the vulvodynia, both of which helped only temporarily. Approximately 6 months prior to her osteopathic referral, she also started amitriptyline $10 \mathrm{mg}$ daily, which was noted to be ineffective and discontinued. Two months before referral, she started gabapentin $300 \mathrm{mg}$ taken 3 times daily, which helped, but made her feel dull and tired She stated that she felt depressed for several weeks prior to presentation.

The patient was an accountant, married, and stated that her husband was kind and supportive. She was nulliparous and had no past medical, surgical, or relevant family history. Medications included birth control, 
multivitamin, and gabapentin. She denied tobacco, alcohol, or illicit drug use.

The patient's physical examination was normal, with the structural examination revealing SDs that included: sphenobasila symphysis compression; C2 ERrSr; T6-T12 N SrR1, T12FRISl; paraspinal hypertonicity of left greater than right thoracolumbar junction; left SI joint restriction with decreased primary respiratory mechanism; left greater than right suprapubic fascial tension; left pelvic floor inhaled, with severely limited and delayed movement when compared with thoracic respiration; left innominate posterior, outflared; left lower limb functionally short; left gluteus medius tenderness; left hip externally rotated; left hamstring hypertonicity; left lower ribs inhaled; left hemidiaphragm inhaled.

OMT was applied to the affected areas using balanced ligamentous tension, myofascial release, and osteopathy in the cranial field. Most attention was given to the pelvic diaphragm to amplify and coordinate its movement to the thoracic outlet diaphragmatic breathing. With the patient supine with a bolster under the knees, the physician's (A.G.) caudad hand contacted the medial border of the ischial tuberosity, applying a very gentle, cephalad-lateral balanced tension. The cephalad hand was placed on the left 11th and 12 th ribs and applied a balanced lateral traction to engage the thoracic diaphragm. To manage the suprapubic tension, one hand contacted the sacrum and the other was placed anteriorly on either side of the suprapubic midline to lift with balanced tension and soften the fascia and balance the uterine and bladder ligaments. The pelvic SDs were corrected using BLT at the SI joint, which resulted in the correction of the left functionally short lower limb. She was advised not to wear the heel lift.

On the first follow-up visit, she stated the vulvodynia had lessened, but her left hip and hamstring pain was more noticeable. Sitting was less painful. There was decreased restriction in the pelvic floor, but the left side was still more hypertonic than the right. The diaphragms were moving more synchronously, and her lower limbs were even. An MRI of the left hip was ordered and revealed a high signal in the region of the left hamstring tendons' insertion, with a subtle high signal in the adjacent soft tissues, reflecting tendinosis or partial thickness tearing. There was no labral tear or hip effusion. On 3 subsequent weekly visits, the vulvodynia continued to lessen in severity. The patient's symptoms of depression and pain both improved. After 3 monthly visits, the patient was feeling significantly better and weaned off gabapentin.

\section{Discussion}

Management options for vulvodynia often start with behavioral modifications, such as vulvar hygiene, eliminating skin irritants, soaking in warm baths, ice packs, and topical lubricants to reduce any exacerbating factors. ${ }^{3}$ Management also includes home exercises and pelvic floor physical therapy. Topical medications, such as lidocaine or hormonal or capsaicin creams, have been used with inconsistent results. There is limited supporting data regarding the use of oral medications, such as antidepressants, anticonvulsants, or muscle relaxants. More invasive managements include steroid or Botox injections; surgery or laser treatments may be considered for vulvodynia as a last resort. Counseling with a mental health professional may be considered when treating patients with vulvodynia.

Regional structural interdependence of the pelvis to the hip and the biceps femoris is a potential etiology or a contributor to perineal pain. The patient likely injured the hamstring attachment on the left ischial tuberosity during the long walk that preceded her pain onset. This injury may have strained the sacrotuberous ligament, altering pelvic and SI joint mechanics. Dysfunctional SI joint mechanics alter the tension on the pelvic floor. These regional SDs may have led to the vulvodynia. While not commonly used for perineal pain, OMT should be considered part of the multimodal treatment approach applied to cases of vulvodynia. If contributing somatic dysfunctions are identified and managed, vulvodynia may be improved or resolved 
completely. Drawing on our tenets, osteopathic physicians are uniquely qualified to provide a whole-person approach to patients with vulvodynia throughout the clinical encounter, maintaining open communication and ensuring informed consent to optimize patient comfort and treatment outcomes. Future studies using OMT for vulvodynia are needed to show the benefits of this management. Overall, maximal symptom reduction often requires a variety of treatment modalities.

\section{Conclusion}

Vulvodynia is a complicated diagnosis where patients are often left suffering from a variety of ineffective treatment modalities. Repercussions of this disorder can have a negative effect on a woman's health, as well as her intimate relationships, social activities, selfesteem, and work productivity. Treatment needs to be individualized to each patient's needs. In this case, OMT, a nonpharmacological, noninvasive therapy, was found to be effective in reducing perineal pain.

\section{Author Contributions}

All authors provided substantial contributions to conception and design, acquisition of data, or analysis and interpretation of data; all authors drafted the article or revised it critically for important intellectual content; all authors gave final approval of the version of the article to be published; and all authors agree to be accountable for all aspects of the work in ensuring that questions related to the accuracy or integrity of any part of the work are appropriately investigated and resolved.

\section{References}

1. Loflin BJ, Westmoreland K, Williams NT. Vulvodynia: a review of the literature. J Pharm Technol. 2019;35(1):11-24. doi:10.1177/ 8755122518793256

2. Harlow BL, Kunitz CG, Nguyen RH, Rydell SA, Turner RM, MacLehose RF. Prevalence of symptoms consistent with a diagnosis of vulvodynia: population-based estimates from 2 geographic regions. Am J Obstet Gynecol. 2014;210(1):40.e41-48.
3. Eickmeyer SM. Anatomy and physiology of the pelvic floor. Phys Med Rehabil Clin N Am. 2017;28(3):455-460. doi:10.1016/j. ajog.2013.09.033

4. Bornstein J, Goldstein AT, Stockdale CK, et al. 2015 ISSVD, ISSWSH and IPPS consensus terminology and classification of persistent vulvar pain and vulvodynia. Obstet Gynecol. 2016;127(4):745-751. doi:10.1097/AOG.0000000000001359

5. Edwards L. Vulvodynia. Clin Obstet Gynecol. 2015;58(1):143-152. doi:10.1097/GRF.0000000000000093

6. Tettambel MA. An osteopathic approach to treating women with chronic pelvic pain. J Am Osteopath Assoc. 2005;105(suppl 4):S20-S22.

7. Travaline JM, Ruchinskas R, D’Alonzo GE, Jr. Patient-physician communication: why and how. J Am Osteopath Assoc. 2005;105 (1):13-18.

8. Conigliaro R, Kuperstein J, Dupuis J, et al. The PEEER model: effective healthcare team-patient communications. MedEdPORTAL Publications. 2013;9. doi:10.15766/mep_2374-8265.9360

9. DeRosa C, Porterfield JA. Anatomical linkages and muscle slings of the lumbopelvic region. In: Movement, Stability \& Lumbopelvic Pain. Elsevier Ltd; 2007:47-62.

10. Williams A, Newell R, Davies M, Collins P. Pelvic girdle and lower limb. In: Standring S, ed. Gray's Anatomy The Anatomical Basis of Clinical Practice. 39th ed. Elsevier Churchill Livingstone; 2016:1419-1459.

11. Williams A, Newell R, Collins P. Back and macroscopic anatomy of spinal cord. In: Standring S, ed. Gray's Anatomy The Anatomical Basis of Clinical Practice. 39th ed. Elsevier Churchill Livingstone; 2016:733-773.

12. Standring S, Williams A. True pelvis, pelvic floor and perineum. In: Standring S, Gray's Anatomy The Anatomical Basis of Clinical Practice 39th ed. Elsevier Churchill Livingston; 2016:1357-1371.

13. Fitzgerald MP, Kotarinos R. Rehabilitation of the short pelvic floor. II: Treatment of the patient with the short pelvic floor. Int Urogynecol J Pelvic Floor Dysfunct. 2003;14(4):269-275. doi:10.1007/ s00192-003-1050-7

14. Snijders CJ, Vleeming A, Stoeckart R. Transfer of lumbosacral load to iliac bones and legs part 1: Biomechanics of self-bracing of the sacroiliac joints and its significance for treatment and exercise. Clin Biomech. 1993;8(6):285-294.

15. Bierry G, Simeone FJ, Borg-Stein JP, Clavert P, Palmer WE. Sacrotuberous ligament: relationship to normal, torn, and retracted hamstring tendons on MR images. Radiology. 2014;271(1):162-171. doi:10.1016/0268-0033(93)90002-Y

16. Tettambel M, Nelson, K. The female patient. In: Nelson K, Glonek T, ed. Somatic Dysfunction in Osteopathic Family Medicine. 2nd ed. Wolters Kluwer Health; 2015.

17. Willard F. Autonomic nervous system. In: Seffinger M, ed. Foundations of Osteopathic Medicine. 4th ed.: Wolters Kluwer; 2018:221-267.

๑) 2020 American Osteopathic Association 